Annuaire suisse de politique de développement

$17 \mid 1998$

Propriété intellectuelle : quels enjeux pour les pays en développement ?

\title{
Les défis lancés par l'accord TRIPS aux pays en développement
}

Nadine Keim

\section{CpenEdition}

\section{Journals}

Édition électronique

URL : http://journals.openedition.org/aspd/753

DOI : $10.4000 /$ aspd.753

ISSN : 1663-9669

Éditeur

Institut de hautes études internationales et du développement

Édition imprimée

Date de publication : 1 avril 1998

Pagination : 65-72

ISSN : $1660-5934$

Référence électronique

Nadine Keim, «Les défis lancés par l'accord TRIPS aux pays en développement », Annuaire suisse de politique de développement [En ligne], 17 | 1998, mis en ligne le 30 juillet 2012, consulté le 08 septembre 2020. URL : http://journals.openedition.org/aspd/753; DOI : https://doi.org/10.4000/aspd.753 


\section{LES DÉFIS LANCÉS PAR L'ACCORD TRIPS AUX PAYS EN DÉVELOPPEMENT}

NADINE KEIM COMTE*

A VEC l'Organisation mondiale du commerce (OMC), les pays du Sud n'ont plus la possibilité de choisir d'imiter les techniques créées par d'autres. Au-delà de quelques exceptions, l'accord TRIPS prévoit la protection des inventions technologiques par un brevet. En outre, il oblige à breveter les micro-organismes, ainsi qu'à protéger d'une façon ou d'une autre les variétés végétales. Dorénavant, la seule propriété intellectuelle au monde non protégée, ce sont les connaissances traditionnelles du Sud. Résultat: le système des brevets, reconnu et bien réel, s'oppose à d'éventuelles compensations selon le bon vouloir des pays riches. Pour les pays en développement, cela est synonyme d'obligations administratives et de coûts supplémentaires. C'est aussi un véritable défi législatif et éthique.

Avec un brevet, l'inventeur possède un droit d'exclusivité commerciale pour son invention, limité dans le temps, en échange de la publication immédiate de ses secrets. C'est un compromis entre les compensations dues à un inventeur privé et le bien-être de la société en général afin de permettre de nouvelles découvertes. Conçu pour promouvoir la recherche et l'industrialisation, le brevet devient de plus en plus un instrument de monopole et de contrôle au bénéfice des industries technologiques avancées du Nord. En fait, environ $90 \%$ des brevets mondiaux sont délivrés dans le Nord et plus de la moitié de ceux déposés dans le Sud sont détenus par des étrangers.

Une protection trop stricte de la propriété intellectuelle peut freiner le développement économique d'un pays. Mais une fois «industrialisé», celui-ci devient un farouche défenseur du brevet. Avec l'apparition de la biotechnologie et du génie génétique, le développement d'une invention coûte de plus en plus cher, alors que sa copie reste relativement simple, rapide et bon marché. L'importance de la propriété intellectuelle va se renforcer, notamment en raison de l'intensification des échanges, mais aussi de la peur croissante du Nord de perdre sa domination des marchés et sa compétitivité.

\section{LA SUISSE S'EST DÉVELOPPÉE EN PIRATANT LES INVENTIONS ÉTRANGÈRES}

Au siècle dernier, de nombreux industriels suisses ont combattu la protection de la propriété intellectuelle. La première loi dans ce domaine n'est entrée en vigueur qu'en 1888 . Notre pays a subi, pendant des décennies, une réputation internationale de pays pirate, l'industrie chimique n'hésitant jamais à imiter les produits et les procédés de concurrents étrangers. En 1904, par exemple, Berlin dénonçait le pillage helvétique des inventions allemandes, telles que l'aspirine

* Coordinatrice de politique de développement à la Communauté de travail Swissaid/Action de Carême/Pain pour le prochain/Helvetas/Caritas, Lausanne. 
ou l'hérö̈ne. Les entreprises s'épargnaient ainsi les frais de recherche et développement tout en vendant leurs copies meilleur marché que les originaux. Sans cela, le passage à une industrie pharmaceutique compétitive aurait été autrement plus difficile et onéreux. La protection des médicaments, en tant que produits finis, n'est intervenue en Suisse qu'en 1978'.

La protection des inventions par les brevets n'est pas un facteur clé du développement économique. Aucune progression d'un pays ne l'a jamais prouvé. Au contraire, le Japon, la Corée ou Taiwan ont connu des succès économiques en copiant les inventions étrangères, ce qui était alors possible avec l'absence d'un système moderne de brevets. Les brevets sont à l'extrême opposé des idées, actuellement en vogue, de concurrence et d'économie de marché. Les penseurs libéraux des débuts étaient conscients de cette contradiction. Au siècle passé, le succès des défenseurs des brevets représentait la victoire des protectionnistes. L'on parlait alors d'un droit naturel à l'imitation.

\section{LE SUD N'A PLUS LE CHOIX}

Un produit breveté dans un pays n'est, en règle générale, pas protégé dans les Etats voisins. C'est pourquoi les pays cherchent de plus en plus à coopérer entre eux. Ils ont débuté en 1883 avec la Convention de Paris. Cette dernière, ainsi que des accords ultérieurs, sont gérés aujourd'hui par l'OMPI (Organisation mondiale de la propriété intellectuelle), mais les pays développés ne les jugent plus assez contraignants. L'accord TRIPS (Accord sur la protection de la propriété intellectuelle liée au commerce) représente les premières normes contraignantes harmonisées à l'échelle planétaire dans ce domaine (art. 27.1 et 33). Il est l'un des principaux piliers de l'OMC (Organisation mondiale du commerce), entrée en vigueur le $1^{\text {er }}$ janvier 1995 et dont plus de 130 pays sont membres.

L'accord TRIPS oblige les pays à protéger les inventions technologiques par un brevet pendant au moins vingt ans. L'invention doit être nouvelle, impliquer une activité inventive ou non évidente et être susceptible d'application industrielle ou utile. L'application de cet accord en général sera examinée par l'OMC tous les deux ans dès le $1^{\text {er }}$ janvier 2000 (art. 71). Résultat: les pays du Sud n'ont plus, contrairement aux pays riches à l'époque de leur industrialisation, la possibilité de choisir d'imiter ou d'adapter les techniques créées par d'autres. Dorénavant, les pays pauvres importateurs de technologies sont même obligés de payer des licences sur certains produits alimentaires ou certains médicaments jugés essentiels.

\section{FLEXIBILITÉ, MAIS SURTOUT DES COÛTS}

Des périodes transitoires sont néanmoins prévues pour les pays en développement. Ils ont cinq ans à partir de l'entrée en vigueur de l'OMC, c'est-à-dire jusqu'au $1^{\text {er }}$ janvier 2000, pour mettre en œuvre les normes minimales en matière de brevet. En outre, pour les inventions dans le domaine pharmaceutique et

1. Ordonnance fédérale sur les brevets d'invention du 19 octobre 1977. 
agrochimique, il est prévu une période additionnelle de cinq ans (art. 65), bien que des droits exclusifs de commercialisation doivent être accordés dès le départ (art. 70). Pour les pays les moins avancés, l'échéance a été fixée au $1^{\text {er }}$ janvier 2006 et des prorogations sur demande sont possibles au-delà (art. 66).

Au-delà des périodes transitoires, l'accord TRIPS offre, sur papier, quelques exceptions qui devraient permettre aux pays du Sud d'agir selon leurs propres intérêts de développement. Premièrement, il prévoit que les pays membres peuvent prendre des mesures «pour promouvoir l'intérêt public dans des secteurs d'une importance vitale pour leur développement socio-économique et technologique» (art. 8). Ensuite, une protection par brevet n'est pas obligatoire, si cela est «nécessaire» pour éviter une atteinte à l'ordre public et aux bonnes mœurs, à la santé et la vie des personnes et des animaux, ainsi qu'aux végétaux ou à l'environnement (art. 27.2). Par ailleurs, le droit des chercheurs d'utiliser des inventions brevetées, sans frais, pour effectuer des recherches non commerciales est garanti (art. 30).

Dans certains cas, les pays peuvent aussi utiliser un brevet sans le consentement de son titulaire (art. 31). Il s'agit de ce que l'on appelle les «licences obligatoires ». L'accord TRIPS n'en limite pas l'utilisation, mais les soumet à des conditions sévères. Il serait, par exemple, possible pour un pays de décréter une licence obligatoire en cas d'intérêt public, de santé publique, d'urgence nationale, d'utilisation publique à des fins non commerciales ou d'abus monopolistique. Dans le même ordre d'idées, l'accord TRIPS permet aux pays de prendre des mesures nationales pour restreindre les pratiques anticoncurrentielles des entreprises privées (art. 40). En pratique, l'avenir dira si les pays pauvres peuvent utiliser ces possibilités d'exception et surtout s'ils peuvent les défendre au sein de l'OMC en cas de litiges. Il est clair que seuls les pays dotés d'une administration forte et spécialisée peuvent faire valoir leurs droits.

Pour les pays en développement, les contraintes de l'OMC sont surtout synonymes d'obligations administratives accrues. Rien que pour le Bangladesh, les coûts supplémentaires de la mise sur pied de normes en matière de brevets sont estimés à plus d'un million de dollars par an. Cela implique, notamment, l'élaboration d'une loi, la création d'une administration, la formation d'experts et la défense des intérêts du pays au sein de l'OMC. Par conséquent, l'accord TRIPS est un formidable défi législatif pour les pays du Sud. Ils dépendent beaucoup de la promesse de coopération technique que leur ont faite les pays occidentaux, notamment dans l'élaboration des lois, la prévention des abus, le renforcement des bureaux et la formation du personnel (art. 67), ainsi que dans le transfert de technologie (art. 66).

L'OMPI a, jusqu'à ce jour, assuré un rôle de coopération technique avec les pays en développement dans le domaine de la propriété intellectuelle. Des conseils, une formation et du matériel sont ainsi mis à leur disposition. Mais cela ne suffit plus. Les pays industrialisés doivent contribuer davantage, bilatéralement et au travers d'organismes multilatéraux compétents, à faire en sorte que le Sud puisse être en mesure d'élaborer une législation conforme à leurs intérêts de développement. Une des principales productrices de brevets par tête d'habitant au monde, la Suisse est tout particulièrement interpellée à ce sujet. Elle s'est battue, lors des négociations de l'accord TRIPS, aux côtés des EtatsUnis et de l'Union européenne contre la plupart des pays en développement. 


\section{LA PORTE S'OUVRE AU BREVET SUR LA VIE}

Un des sujets les plus controversés, lors des négociations de l'accord TRIPS, était l'extension de la brevetabilité aux organismes vivants. Finalement, les pays membres de l'OMC se sont ralliés à un compromis, qui reflète l'interprétation de la législation dans les pays occidentaux. Les micro-organismes et les procédés microbiologiques et techniques doivent être brevetés (art. 27.3b). Par exemple, les pays sont tenus de protéger par brevet une bactérie modifiée génétiquement. L'accord TRIPS rend ainsi explicitement possible la protection du vivant. Mais il n'oblige pas à breveter les plantes, les animaux et les procédés naturels. Néanmoins, il a été convenu de prévoir «la protection des variétés végétales par des brevets, par un système sui generis efficace, ou par une combinaison de ces deux moyens» (art. 27.3b).

En d'autres mots, s'ils ne sont pas obligés de breveter les inventions végétales, les pays sont, par contre, tenus de les protéger par un autre système de leur choix. Les négociateurs avaient pensé à la Convention UPOV (voir plus loin), mais rien dans le texte ne s'oppose à ce qu'il s'agisse d'un système autonome. Le «hic», c'est que la possibilité de mettre sur pied un tel système dans l'intérêt du pays et de son développement risque de disparaître avant même d'avoir vu le jour. D'une part, l'OMC a pour tâche de réexaminer l'article $27.3 \mathrm{~b}$ concernant cette possibilité d'ici au $1^{\text {er }}$ janvier 1999. De l'autre, cette même disposition n'exige pas avant le $1^{\text {er }}$ janvier 2000 la mise en vigueur d'une protection des variétés végétales par un système sui generis efficace.

Il n'existe actuellement pas d'autres modèles de protection des variétés végétales, en dehors des brevets ou de l'UPOV. La tâche n'est pas aisée pour les pays du Sud, dans la mesure où la signification de sui generis et plus encore d' «efficace» n'est pas clairement définie. Il reste beaucoup à faire pour prouver aux pays occidentaux qu'il est possible de mettre sur pied d'autres systèmes. L'Inde, la Thaillande et les Philippines ainsi que les pays du Pacte andin se sont jetés à l'eau. Ils élaborent activement des législations autonomes. Méfiants, les Etats-Unis les suivent très attentivement. En Thaîlande, par exemple, les diplomates américains ne se gênent pas d'émettre déjà maintenant leurs critiques!

On le voit, limité à l'origine aux inventions physiques, le brevet est en train de s'élargir sournoisement aux organismes vivants. Les plantes et les animaux deviennent ainsi de plus en plus la propriété intellectuelle d'intérêts privés. Dorénavant, la seule propriété intellectuelle au monde qui n'est pas protégée recouvre les connaissances traditionnelles des gens du Sud. C'est une inégalité fondamentale, qui pose deux problèmes supplémentaires aux pays en développement.

\section{PRIVILĖGES DES PAYSANS: DE PLUS EN PLUS BAFOUÉS}

Encore aujourd'hui, l'échange traditionnel entre les paysans pauvres du Sud représente près de $80 \%$ des besoins en semences. Cela leur permet de limiter leurs coûts et leur dépendance à l'égard de fournisseurs extérieurs. Cette pratique est actuellement remise en question par la protection de la propriété intellectuelle. Ce qui était depuis toujours un droit inaliénable est devenu, dans les 
années 80 , un «privilège» sujet aux humeurs politiques. Si on le lui retire, un agriculteur cultivant un maïs modifié est désormais tenu de payer des redevances à chaque fois qu'il désire garder quelques épis comme semence pour la récolte suivante.

Pour les plantes, il existe depuis 1961 la Convention internationale pour la protection des obtentions végétales (UPOV). A la différence des brevets, ce système de protection garantissait, à son origine, le libre accès aux inventions améliorées à des fins non commerciales, sans qu'un droit doive être payé. Premièrement, les paysans étaient libres de réutiliser et d'échanger les semences d'une variété protégée pour la prochaine récolte ("privilège de l'agriculteur»). Deuxièmement, l'utilisation d'une variété protégée pour la création d'une nouvelle variété et l'exploitation commerciale de cette dernière étaient possibles (« réserve de l'obtenteur»). Mais la dernière révision de l'UPOV en 1991 a fortement gommé ces avantages. Pire, le «privilège de l'agriculteur» a été laissé au bon vouloir des lois nationales. Autre problème majeur: la nouvelle Convention UPOV ne protège pas les semences traditionnelles des paysans, sous prétexte qu'elles ne sont pas nouvelles et qu'elles ne sont pas stables (elles peuvent se modifier en se reproduisant). Dans bien des cas, ces variétés répondent pourtant aux besoins de ceux qui les ont sélectionnées et continuent de le faire aussi bien sinon mieux que celles provenant de la recherche formelle.

Seuls huit pays du Sud - sur 34 - sont membres de la Convention de l'UPOV version 1978, alors que la dernière version de 1991 n'est pas encore en vigueur. Le Secrétariat de l'UPOV tente actuellement de faire le tour des pays du Sud en faisant de la propagande pour la nouvelle mouture. Celle-ci n'est cependant pas obligatoirement la meilleure solution pour ceux qui souhaitent reconnaître le "privilège des paysans" et les variétés traditionnelles. A tout choisir, la Convention de 1978 doit être préférée à celle de 1991 (cela est possible aussi longtemps que celle de 1991 n'est pas entrée en vigueur).

\section{DROITS DES PAYSANS: TOUJOURS THÉORIQUES}

Les plantes et les animaux sauvages du Sud sont aujourd'hui les nouvelles matières premières à la mode. Même si elles sont basées sur des produits naturels, les «inventions » des communautés locales ne se trouvent pas telles quelles dans la nature. Elles sont le produit du savoir humain. Pour transformer une plante en un médicament, il faut connaître l'espèce en question, sa localisation, la partie à utiliser, la manière de la préparer, les ingrédients à rajouter, la posologie, etc.

Le régime actuel de la propriété intellectuelle est ainsi fait qu'il permet aux multinationales d'acquérir les innovations indigènes sans leur accorder la moindre reconnaissance. Tandis que les apports des communautés du Sud représentent l'essentiel de la biodiversité de notre planète, ce sont les multinationales du Nord qui s'y approvisionnent librement et sans bourse délier. Elles peuvent alors déposer un brevet sur les plantes modifiées et les vendre à prix fort, quelquefois au pays d'origine de ces plantes. Cela occasionne, selon les estimations des Nations Unies, un manque à gagner de plus de 5 milliards de dollars par an en licences non versées aux pays du Sud. 
En 1983, les pays membres de la FAO ont mis sur pied l'Engagement international sur les ressources phytogénétiques, un accord volontaire entre plus de 100 pays pour préserver et utiliser les plantes. Le principe: les ressources génétiques végétales sont un héritage de l'homme et doivent donc être disponibles sans restriction (art. 1). En 1989, une annexe a été rajoutée, qui reconnaît les «droits des paysans », c'est-à-dire les contributions passées, présentes et futures dans la conservation, l'amélioration et l'accès aux ressources génétiques végétales (Annexe II).

\section{DROIT CONTRE PROMESSE}

Peu a été accompli depuis pour concrétiser les "droits des paysans». Il est notamment difficile de donner une valeur monétaire «équitable» à la contribution ou au savoir des communautés locales. Autre difficulté: à qui et sous quelle forme cette compensation doit-elle être versée? Finalement, la notion même de propriété diffère parfois fondamentalement entre les pays occidentaux et les sociétés traditionnelles. Dans les pays du Sud, la propriété n'est souvent pas individuelle mais commune. Le fonds international de la FAO pour dédommager les droits des agriculteurs est également resté inactif.

Instruments signés lors du Sommet de la Terre à Rio en 1992, la Convention sur la biodiversité et l'Agenda 21 n'ont malheureusement rien clarifié. Contraignante, la première a admis sur papier le principe de la souveraineté nationale sur les ressources biologiques (art. 15). Elle a reconnu le rôle et les droits des sociétés traditionnelles et indigènes à tirer profit de leurs plantes (art. 8). Elle a prévu le partage équitable des bénéfices, sous forme d'accès aux technologies et de participation prioritaire à la recherche pour le Sud (art. 16 et 19). Cependant, la Convention sur la biodiversité reste ambiguë sur l'application de ces principes, notamment en relation avec les droits de propriété intellectuelle. La Suisse, les Etats-Unis et l'Union européenne l'ont signée sous réserve que les transferts de technologie doivent respecter les brevets.

Ainsi s'opposent toujours d'un côté un système de droit des brevets, reconnu et bien réel, et de l'autre d'éventuelles promesses de compensation selon le bon vouloir des pays riches. Les divergences sont toujours entières entre les pays qui soutiennent un système simple de compensation et ceux qui souhaitent une véritable législation sur les «droits des paysans» :

- en tant que cultivateurs, droit de réutiliser les semences protégées pour la prochaine récolte, ainsi que de les échanger;

- en tant que détenteurs de ressources, droit de contrôler les plantes et le savoir, ainsi que d'accéder aux résultats des recherches;

- en tant qu'acteurs de la conservation et de l'amélioration de la diversité biologique, droit d'être reconnus pour leurs contributions, ainsi que de recevoir une part juste des bénéfices. 


\section{A LA RECHERCHE DE DROITS POUR L'INNOVATION COMMUNAUTAIRE}

Le brevet, créé il y a plus d'un siècle par les Occidentaux pour protéger leurs inventions de machines, n'est pas adapté pour protéger la contribution intellectuelle des communautés indigènes. Les "privilèges» et «droits des paysans» sont en fait un droit inédit, qui récompense non pas une invention nouvelle, mais une conservation et une tradition. Perçu en Occident comme une contradiction même de la notion de "propriété intellectuelle», ce nouveau droit est en fait complémentaire. Par conséquent, les pays du Sud se retrouvent face à un défi de taille: élaborer un système différent de propriété intellectuelle, qui offre aussi une place aux droits communautaires. Ce système pourrait, par exemple, s'inspirer des modèles suivants:

- Les «dispositions types sur le folklore» de l'UNESCO/OMPI de 1985 comportent trois éléments novateurs, notamment adaptés à la protection des plantes. Les collectivités (au lieu d'individus) peuvent être légalement enregistrées comme inventeuses. Les créations communautaires peuvent être évolutives (non finalisées) tout en restant protégées. Les collectivités conservent le contrôle exclusif sur leur patrimoine aussi longtemps qu'elles continuent à innover.

- Le projet de «Déclaration des Nations Unies sur les droits des peuples indigènes » de 1994 en appelle à reconnaître le savoir, les cultures et les pratiques indigènes. Il leur donne le droit de posséder des ressources (art. 26), ainsi que celui de protéger leurs sciences, y compris les ressources génétiques, les semences, les médecines et le savoir (art. 29).

- La «Convention 169 concernant les peuples indigènes et tribaux» de l'OIT de 1989 reconnaît les droits de propriété des peuples indigènes sur les terres qu'ils occupent traditionnellement (art. 14). Elle garantit leurs droits sur les ressources naturelles dont sont dotées leurs terres; droits qui comprennent celui de participer à l'utilisation, à la gestion et à la conservation de ces ressources (art. 15).

\section{LES DÉFIS À RELEVER}

Dans un récent document, la Communauté de travail Swissaid/Action de carême/Pain pour le prochain/Helvetas/Caritas présente des réflexions sur le thème des brevets du point de vue du développement du Sud et propose notamment les revendications suivantes:

- Les pays du Sud doivent profiter des années de transition dont ils disposent dans l'accord TRIPS pour élaborer leur législation sur les brevets.

- Les pays du Sud doivent profiter des flexibilités de l'accord TRIPS pour défendre au mieux leurs intérêts de développement: exceptions pour cause d'ordre public, licences obligatoires, mesures en cas de pratiques anticoncurrentielles.

- Les pays du Sud doivent obtenir les promesses de coopération technique et financière, formulées par le Nord, pour être en mesure d'élaborer une législation conforme à leurs intérêts de développement et de mieux se défendre 
au sein de l'OMC (notamment en vue de la révision de l'article 27, 3 b prévue en 1999 et de celle de l'accord TRIPS en général dès 2000).

- Les pays du Sud ne doivent pas être forcés à adopter un régime de brevet sur la vie. Ils doivent rester libres d'adopter des démarches autonomes différentes (sui generis) ou supplémentaires («privilèges» et «droits des paysans »).

ป Le «privilège des paysans» de réutiliser les semences d'une variété protégée pour sa prochaine récolte doit rester un droit inaliénable. En parallèle, le rôle des semences traditionnelles doit être mis en valeur.

a Les inventions informelles des communautés locales du Sud doivent pouvoir être protégées. Les «droits des paysans» de la FAO, ainsi que le fonds international dans ce domaine, doivent être concrétisés. L'Engagement de la FAO, une fois revu et précisé, doit devenir un instrument international contraignant (annexé en tant que protocole à la Convention sur la biodiversité).

\section{SOURCES}

Bunders J./Haverkort B./Hiemstra W., Biotechnology: Building on Farmers'Knowledge, Macmillan, London, 1996.

Correa C./WHO, The Uruguay Round and Drugs, Geneva, 1997.

Cosbey A./IISD, The Substainable Development Effects of the WTO TRIPs Agreement, Winnipeg, 1996.

Gerster R., Patentierte Profite: zur Rolle schweizerischer Patente in der Dritten Welt, Z Verlag, Basel, 1980.

Keim N./Communauté de travail Swissaid/Action de carême/Pain pour le prochain/Helvetas/Caritas, «Brevet sur la vie? », Revue Sud 3/octobre 1997, Berne/Lausanne.

Lesser W./UNEP, Institutional Mechanisms supporting Trade in Genetic Materials, Geneva, 1994.

Singh Nijar G./TWN, TRIPs and Biodiversity: A Thirld World View, Penang, 1996.

Ouvrage collectif/CRDI, Un brevet pour la vie, Ottawa, 1994.

RAFI/UNDP, Conserving Indigenous Knowledge : Integrating Two Systems of Innovation, New York, 1994.

UICN, Guide de la Convention sur la diversité biologique, Gland, 1994.

UNCTAD, The TRIPs Agreement and Developing Countries, Geneva, 1996.

WWF International, The UN Biodiversity Convention and the WTO TRIPs Agreement, Gland, 1995. 